\title{
Editorial
}

\section{GREAT special feature}

The Kuiper Airborne Observatory (KAO) ceased operation roughly two decades ago with the expectation that a replacement would soon become available. That did not happen immediately, although in the meantime several space missions devoted to infrared and/or submillimeter astronomy (ISO, Spitzer, Herschel, WISE, to name a few) have yielded a harvest of interesting results. There have also been successful balloon-borne missions, most prominently BLAST. However, the great advantage airborne observatories have for their missions to observe at wavelengths not accessible from the ground is that they can take advantage of rapid instrumental innovation, and one therefore notes with great pleasure that SOFIA (the Stratospheric Observatory For Infrared Astronomy) is operational and producing scientific results. SOFIA, a joint project of NASA and DLR (Germany's space agency) consists of a 2.7-m telescope in a modified Boeing 747SP aircraft. Flying several times a week at altitudes as high as $13700 \mathrm{~m}$, over a projected 20 year operational lifetime, SOFIA allows routine access to wavelengths that would otherwise be blocked by absorption from the water vapor in the atmosphere.

One of the pioneering achievements of the KAO was the heterodyne work at wavelengths as short as 63 microns (the lowest energy fine-structure transition of atomic oxygen). More recently, the HIFI heterodyne instrument onboard Herschel has produced wideband high-resolution spectra at wavelengths between 158 and 600 microns. Building on the legacy of these missions, new technological advancements, also to yet higher frequencies, have allowed sensitive heterodyne observations in the far-infrared from SOFIA. High-resolution terahertz spectroscopy of atomic and molecular species has been the principal aim for the German REceiver for Astronomy at Terahertz frequencies (GREAT), which was developed by a consortium of German research institutes under the leadership of R. Güsten from the Max-Planck-Institut für Radioastronomie in Bonn. The instrument has now been successfully commissioned and operated during a dozen Early Science flights in 2011. In this special feature of Astronomy and Astrophysics, we present 22 articles reporting on the first astronomical results as well as the technologies used. These show the potential of high-resolution, far-infrared spectroscopy to probe the kinematics, physics, and chemistry of star-forming regions, as well as the detection of interstellar molecular species not observable from the ground, some for the first time ever.

C. M. Walmsley, C. Bertout, F. Combes, T. Forveille, S. N. Shore in collaboration with R. Güsten 